\title{
Multi-Level Halftoning by IGS Quantization
}

\section{Tadahiko Kimoto}

Faculty of Science and Engineering, Toyo University, Kawagoe, Japan.

Email: kimoto@toyo.jp

Received August $19^{\text {th }}, 2013$; revised September $15^{\text {th }}, 2013$; accepted September $24^{\text {th }}, 2013$

Copyright (c) 2013 Tadahiko Kimoto. This is an open access article distributed under the Creative Commons Attribution License, which permits unrestricted use, distribution, and reproduction in any medium, provided the original work is properly cited.

\begin{abstract}
Improved gray-scale (IGS) quantization is a known method for re-quantizing digital gray-scale images for data compression while producing halftones by adding a level of randomness to improve visual quality of the resultant images. In this paper, first, analyzing the IGS quantizing operations reveals the capability of conserving a DC signal level of a source image through the quantization. Then, a complete procedure for producing a multi-level halftone image by IGS quantization that can achieve the DC conservation is presented. Also, the procedure uses the scanning of source pixels in an order such that geometric patterns can be prevented from occurring in the resulting halftone image. Next, the performance of the multi-level IGS halftoning is evaluated by experiments conducted on 8-bit gray-scale test images in comparison with the halftoning by error diffusion. The experimental result demonstrates that a signal level to be quantized in the IGS halftoning varies more randomly than that in the error diffusion halftoning, but not entirely randomly. Also, visual quality of the resulting halftone images was measured by subjective evaluations of viewers. The result indicates that for 3 or more-bit, in other words, 8 or more-level halftones, the IGS halftoning achieves image quality comparable to that by the error diffusion.
\end{abstract}

Keywords: Digital Halftone; Multi-Level Halftone; Improved Gray-Scale Quantization; Error Diffusion; Subjective Testing

\section{Introduction}

Digital halftoning is a technique to re-quantize a digital image to fewer bits while preventing the image appearance from being corrupted by producing continuouslooking tones, that is, so-called halftones [1]. For instance, for being printed with a black-and-white printer, an 8-bit gray-scale image is to be re-quantized to one bit or two possible levels. Thus, halftoning with two levels is still indispensable in printing. Also, halftoning with more than two levels is useful for output devices that can produce many levels, such as electrostatic printers and compact LED displays.

Generally in a halftoning method, some kind of signal is added to each pixel of a source image and then, the resulting signal is re-quantized to fewer bits. These signals determine the resulting image appearance. A variety of halftoning methods to generate the additional signals can yield good halftone quality in the resulting images that have been proposed.

Error diffusion is a well-known halftoning method that can produce halftone images of good quality. In the error diffusion, the additional signals are generated by distrib- uting error signals, which are the difference of a source pixel level and the re-quantized pixel level, by a digital filter. Thus, the resulting halftone quality depends on the digital filter.

The improved gray-scale (IGS) quantization [2] is regarded as a halftoning method where the additional signals are generated from the low-order bits of neighboring pixels. The low-order bits in a natural scene image are generally a random variable depending on the image characteristics, and also, they are more random as the bit orders are lower. Consequently, the additional signals become a random variable with these properties.

In this paper, we consider the use of the IGS quantization for multi-level halftoning. By evaluating the performance of multi-level quantization and the visual quality of the resulting halftone images, a manner of using the IGS halftoning instead of the error diffusion halftoning is investigated.

The rest of this paper is organized as follows: Section 2 defines the IGS halftoning. First, the IGS quantization is formulated on the basis of the literature. From an analysis of the recursion formula, it is proven that the DC 
component of a source image can be conserved through the quantization. Then, a complete algorithm to apply the quantization to gray-scale images while achieving the DC conservation is presented. In Section 3, stochastic performance of IGS halftoning is evaluated through an experiment conducted on 8-bit test images. The extent of randomness of the additional signals is measured and compared between three IGS methods and two error diffusion methods. In Section 4, visual quality of IGS halftone images is evaluated through a subjective testing conducted to measure image quality by the subjective evaluations of human observers. From the result, a number of halftone bits can be yielded as good image appearance as that achieved by error diffusion that is estimated. Section 5 concludes the paper.

\section{IGS Halftoning}

\subsection{Principle of IGS Quantization}

The procedure of the IGS quantization from $M$-bit signals to $N$-bit ones, $1 \leq N<M$, is expressed as follows. Here, we suppose that source pixels are of $M$ bits without stating it clearly in the rest of this paper. Let $L_{n}(v)$ denote the $n$ least significant bits of the binary expression of $v$. Also let $U_{n}(v)$ denote a value that consists of the $n$ most significant bits same as those of $v$ and the other bits of zero. Then, an $M$-bit value $v$ can be expressed as

$$
v=U_{N}(v)+L_{M-N}(v) .
$$

For an $M$-bit pixel value $p_{i}$ in a scanning order, $i=1,2, \cdots, T$, where $T$ is the number of pixels in the image, an $M$-bit value $S_{i}$ is defined by the recursion relation

$$
S_{i}=p_{i}+L_{M-N}\left(S_{i-1}\right)
$$

where $S_{0}$ is supposed to be 0 . To ensure that $S_{i}<2^{M}$, if $p_{i}>\left(2^{N}-1\right) 2^{M-N}$, we use instead of Equation (2)

$$
S_{i}=p_{i} .
$$

The $N$-bit result of quantizing $p_{i}$, denoted by $Q_{N}\left(p_{i}\right)$, is given by the $N$ most significant bits of $S_{i}$. In other words,

$$
Q_{N}\left(p_{i}\right)=U_{N}\left(S_{i}\right) / 2^{M-N} .
$$

Figure 1 shows the relationship between source levels and output levels of the IGS quantizer. In summary, $Q_{N}\left(p_{i}\right)$ is given by the relation

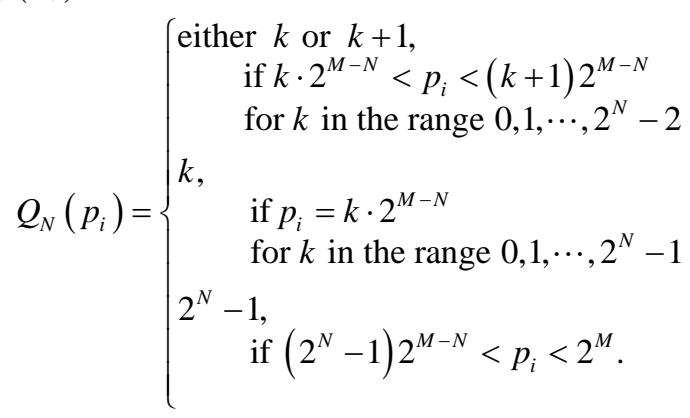

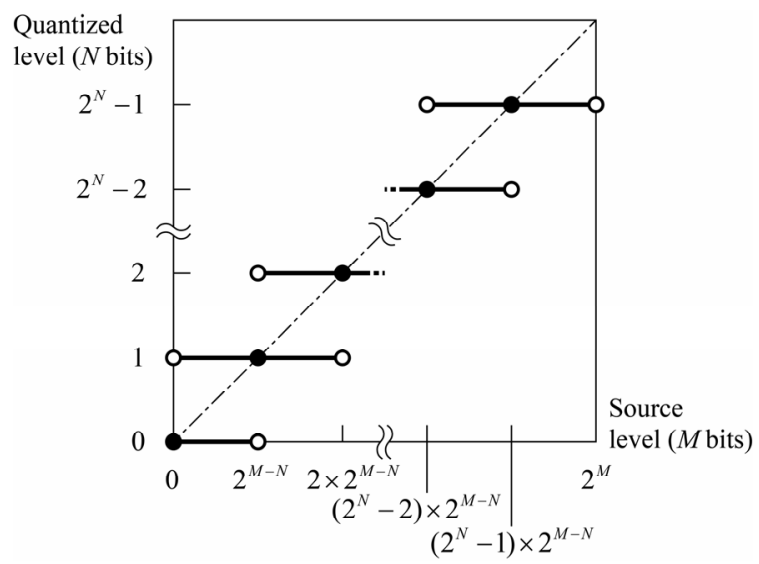

Figure 1. Input-output level relationship of IGS quantizer.

In this relation, for $k \cdot 2^{M-N}<p_{i}<(k+1) 2^{M-N}$ and $0 \leq k \leq 2^{N}-2, Q_{N}\left(p_{i}\right)$ has two possible values, which both depend on $L_{M-N}\left(S_{i-1}\right)$.

\subsection{Mathematical Properties}

A sum of the quantized pixels can be described as follows: Expressing $S_{i-1}$ in the form of Equation (1), then, substituting $L_{M-N}\left(S_{i-1}\right)$ into Equation (2) and using Equation (4) for $U_{N}\left(S_{i-1}\right)$ yield the relation

$$
S_{i}+Q_{N}\left(p_{i-1}\right) 2^{M-N}=S_{i-1}+p_{i} .
$$

The summation of each side of Equation (6) for $i$ from 1 to $T$ yields

$$
S_{T}+\sum_{i=1}^{T-1} Q_{N}\left(p_{i}\right) \cdot 2^{M-N}=\sum_{i=1}^{T} p_{i}
$$

Since $S_{0}=0$ and hence, $Q_{N}\left(p_{0}\right)=0$. Substituting the form of Equation (1) for $S_{T}$ yields the final relation:

$$
L_{M-N}\left(S_{T}\right)+\sum_{i=1}^{T} Q_{N}\left(p_{i}\right) \cdot 2^{M-N}=\sum_{i=1}^{T} p_{i}
$$

Dividing both sides of Equation (8) by $T$ gives

$$
\frac{L_{M-N}\left(S_{T}\right)}{T}+\overline{Q_{N}\left(p_{i}\right)} \cdot 2^{M-N}=\overline{p_{i}}
$$

where $\bar{x}$ represents the average of a sequence of $x$. Because $L_{M-N}\left(S_{T}\right)$ takes a value of at most $2^{M-N}-1$, the first term of the left-hand side of Equation (9) can be neglected for large $T$ 's; thus, we obtain

$$
\overline{Q_{N}\left(p_{i}\right)} \cdot 2^{M-N} \approx \overline{p_{i}} .
$$

This relation means that a level of direct current (DC) of the source image is left almost unchanged in the quantized image. Also, because Equation (8) holds for any $T$, the DC level changes by at the most $\left(2^{M-N}-1\right) / T$ at any pixel during the quantizing of the image. Note that $S_{i}^{\prime} s$ given by Equation (3) are excluded in deriving Equation (8). 


\subsection{Implementing of IGS Halftoning}

1) Source signal range

The DC conservation described above is achieved under the condition that all of $S_{i}^{\prime} s$ are given by Equation (2). This condition can be satisfied by limiting the input signals of the quantizer in the range associated with Equation (2). To limit the source pixel levels in the range, we apply a level transformation that maps the whole $M$ bit range $\left[0,2^{M}-1\right]$ onto the range $\left[0,\left(2^{N}-1\right) 2^{M-N}\right]$ to a source image before carrying out the IGS quantization. For simplicity of implementation, a linear mapping is used in the transformation in this paper.

On the other hand, the level distribution in an image is likely to be distorted due to both the above level transformation and the following IGS quantization. On the assumption both that all the pixel levels in the range $\left[0,2^{M}-1\right]$ occur uniformly in an $M$-bit source image and that $L_{M-N}\left(S_{i-1}\right)$ of Equation (2) takes on equally likely random values, it is derived from stochastic analysis that in the resulting $N$-bit image, both the occurrence frequency of level 0 and that of level $2^{N}-1$ are $1 /\left\{2\left(2^{N}-1\right)\right\}$, and those of the other levels are $1 /\left(2^{N}-1\right)$.

Image areas of pixel levels in the range $\left[\left(2^{N}-1\right) 2^{M-N}, 2^{M}-1\right]$ are painted over in the solid level $2^{N}-1$ after the IGS quantization. Such areas posterized in the highest level may degrade the resulting halftone quality. The above level transformation prevents the posterization from occurring while ensuring that the source level $2^{M}-1$ is always quantized to $2^{N}-1$.

2) Scanning order

The scanning order in an image during the IGS quantization affects the results according to the recursion relation of Equation (2). Such processing dependent on the scanning direction is likely to produce visible artifacts in the resulting image.

Scanning in a more complicated order than the raster order can reduce a relation between the scanning result and the image content [3]. Such scanning is implemented by, for example, the Hilbert path. Figure 2 illustrates an

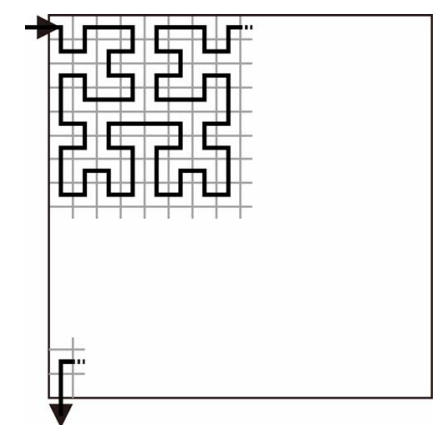

Figure 2. An example of Hilbert scanning path. example of Hilbert path through a squared image with one side of integer-power-of-2 pixels, where the path starts at the upper-left pixel and ends at the lower-left pixel.

Figure 3 shows the system diagram of IGS halftoning process. This diagram involves scanning the whole of a source image once and works in point processing: For every source pixel extracted in the scanning order, first, the level transformation described in the preceding section is carried out and then, the resultant pixel value is quantized by the IGS manner.

\section{Stochastic Performance of IGS Quantization}

\subsection{Measuring Randomness of Additional Signals}

In this section, we evaluate the properties of the additional signals, that is, $L_{M-N}\left(S_{i-1}\right)$ of Equation (2) as a random variable by an experiment with real images. For a given $N$, let $S$ represent a variable that produces $L_{M-N}\left(S_{i}\right)$ for $i=1,2, \cdots, T$ and also, let $s$ be regarded as a random variable in the range $\left[0,2^{M-N}-1\right]$. As measures of randomness of $s$, the following two quantities are used:

1) The memory-less entropy of $\left\{s_{1}, s_{2}, \cdots, s_{T}\right\}$, denoted by $\mu_{N}$, given by

$$
\mu_{N}=-\sum_{s=0}^{2^{M-N}-1} P(s) \log P(s)
$$

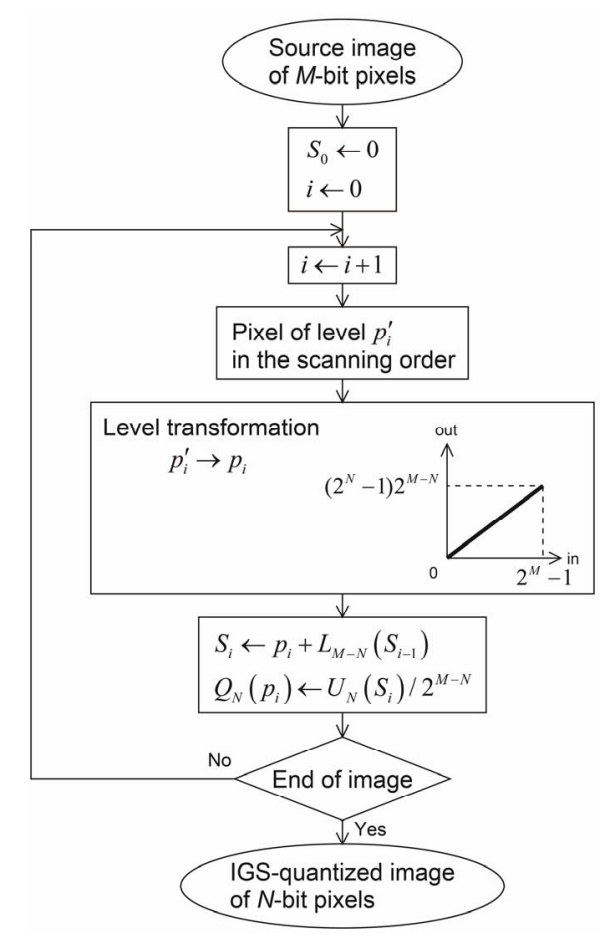

Figure 3. Diagram of IGS halftoning. 
where $P(s)$ is the probability of $s$.

2) The Entropy of $\left\{s_{1}, s_{2}, \cdots, s_{T}\right\}$ conditional on pixels $\left\{p_{1}, p_{2}, \cdots, p_{T}\right\}$, denoted by $v_{N}$, given by

$$
v_{N}=-\sum_{p=0}^{2^{M}-1} P(p) \sum_{s=0}^{2^{M-N}-1} P(s \mid p) \log P(s \mid p)
$$

where $P(s \mid p)$ is the conditional probability that, given that a pixel level is $p$, a signal of level $s$ is added to it, and $P(p)$ represents the occurrence probability of level $p$.

These quantities are compared between the following five methods:

1) Method IGS-RAS implements the IGS quantization by raster scanning.

2) Method IGS-HIL implements the IGS quantization by the Hilbert scanning described in Section 2.3 (2).

3) Method IGS-RND uses an $(M-N)$-bit uniform random number, which is actually generated by a pseudo-random number generator with a computer, instead of $L_{M-N}\left(S_{i-1}\right)$ in Equation (2).

4) Method EDF-FS implements the error diffusion scheme with the error filter proposed by Floyd and Steinberg [4].

5) Method EDF-JJN implements the error diffusion scheme with the error filter proposed by Jarvis, Judice and Ninke [5].

In EDF-FS and EDF-JJN, the error diffusion scheme works to quantize $M$-bit pixels to $N$ bits, $1 \leq N<M$. Because the additional signal that plays the role of $L_{M-N}\left(S_{i-1}\right)$ of Equation (2) takes on fractional values in the error diffusion, itis to be digitized to $M$-bit levels in calculating $\mu_{N}$ and $v_{N}$.

\subsection{Experimental Results}

Experiments of the above methods were conducted on 256-gray scale (that is, $M=8$ ) images with various values of $N$. The source images used in the experiments include vertical and horizontal ramp images shown in Figures 4(a) and (b), respectively, and two natural scene images Barbara and Lena shown in Figures 4(c) and (d), respectively. Note that the image Barbara includes striped regions easy to see, and Lena includes wide smooth regions. For each given $N$, the source images were transformed by the level transformation described in Section 2.3 (1) prior to undergoing any of the halftoning methods.

Figure 5 shows the measurements of the entropy $\mu_{N}$ that were taken in performing each method by varying $N$ for three of the source images. As this figure shows, the entropies in IGS-HIL are almost the same as those in IGS-RND, and consequently, the additional signals in IGS-HIL preserve a nearly uniform randomness for any $N$ as far as the memory-less entropy is concerned.

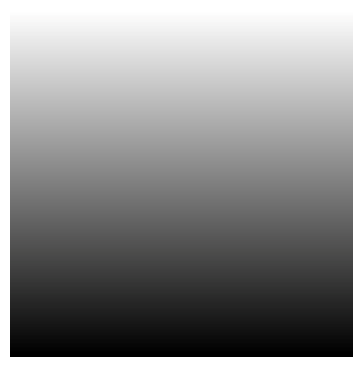

(a)

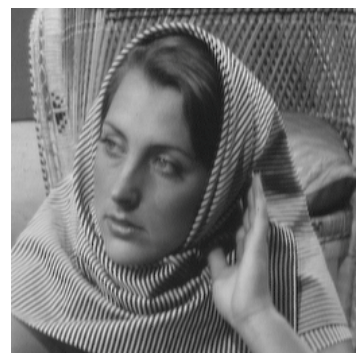

(c)

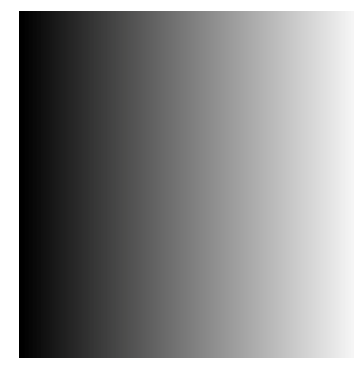

(b)

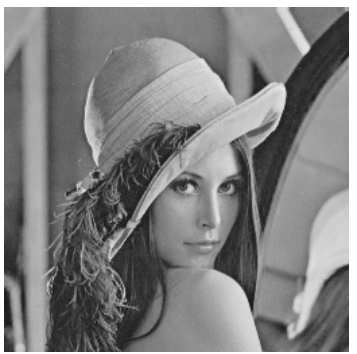

(d)
Figure 4. Examples of 8-bit source images of 256 by 256 pixels used in the experiments: (a) Vertical ramp with 1level per pixel gradation; (b) Horizontal ramp with 1-level per pixel gradation; (c) Barbara and (d) Lena.

Figure 6 shows the measurements of the entropy $v_{N}$ for the three source images. The result indicates that in the three IGS methods, the additional signals in IGS-HIL have more randomness for source pixels than those in IGS-RAS and less randomness than those in IGS-RND, and also, more randomness than those in the two error diffusion methods. In addition, we can observe a difference between the two error diffusion methods due to the error filters; the additional signals are more constrained in EDF-JJN than in EDF-FS.

As regards IGS-RAS, a large difference between the measurements of the entropy of the vertical ramp image and the entropy of the horizontal ramp image is observed from Figure 6. This result demonstrates that the properties of the additional signals in IGS-RAS are apt to depend on the scanning direction.

Figure 7 reveals a visual difference between the IGS results of raster scanning and those of Hilbert scanning. We observe that the IGS quantization yields geometrical patterns from regions of a uniform level gradation through the raster scanning, and the generated patterns depend on the relation between the direction of raster scanning and that of gradation. On the contrary, the Hilbert scanning is effective in inhibiting such patterns from being produced regardless of the direction of gradation.

\section{Visual Quality of Halftones}

\subsection{Subjective Evaluation}

In this section, we consider appearances of multi-level 


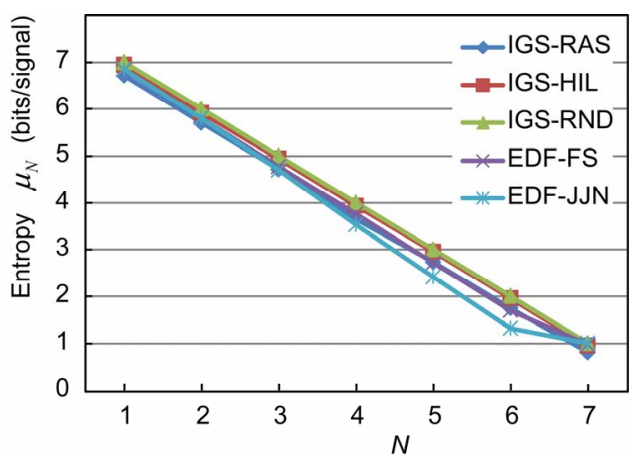

(a)

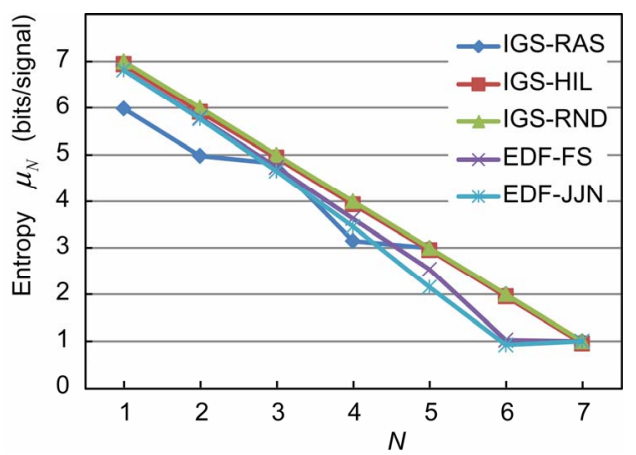

(b)

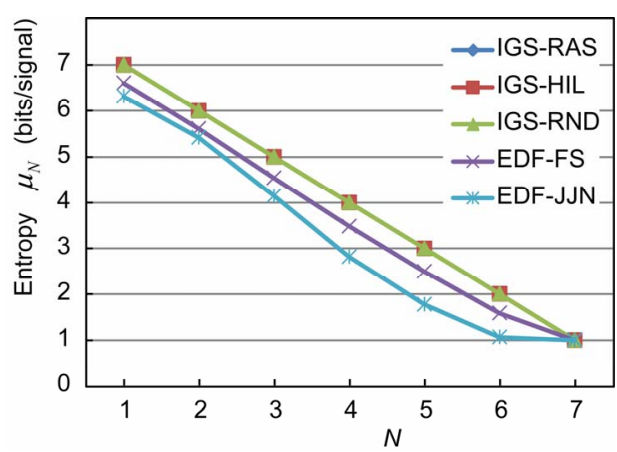

(c)

Figure 5. Entropies $\mu_{N}$ of additional signals in halftoning. (a) The vertical ramp image; (b) The horizontal ramp image; and (c) Barbara.

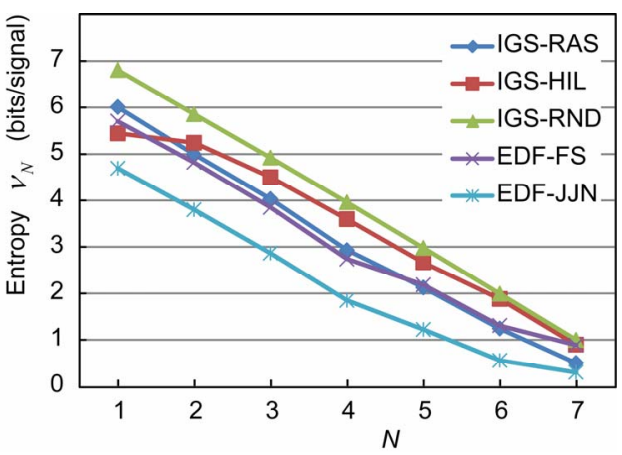

(a)

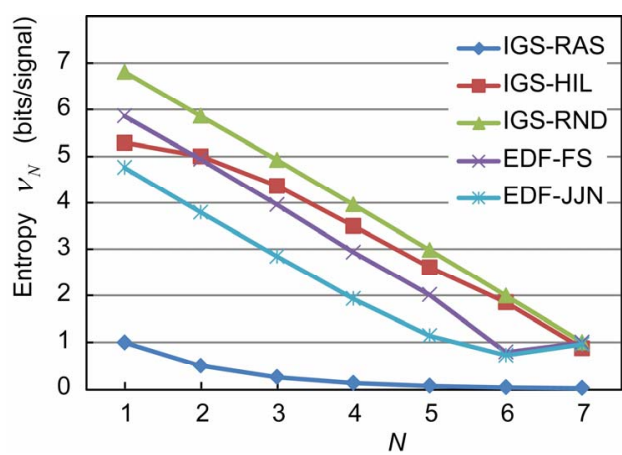

(b)

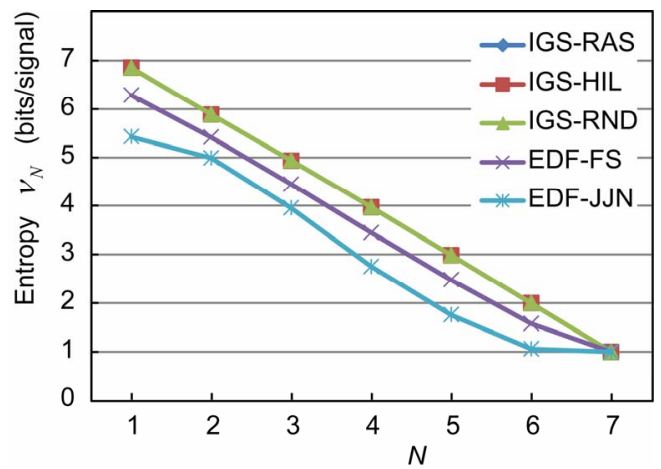

(c)

Figure 6. Entropies $v_{N}$ of additional signalsin halftoning. (a) The vertical ramp image; (b) The horizontal ramp image; and (c) Barbara. 


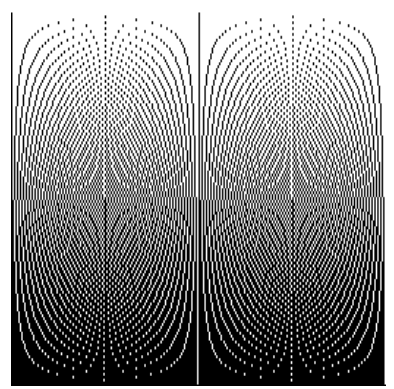

(a)

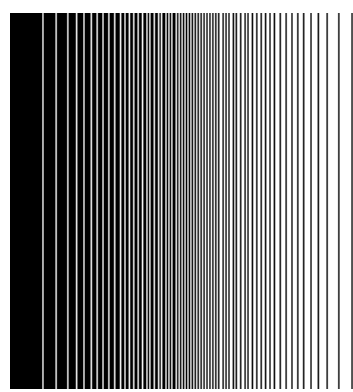

(c)

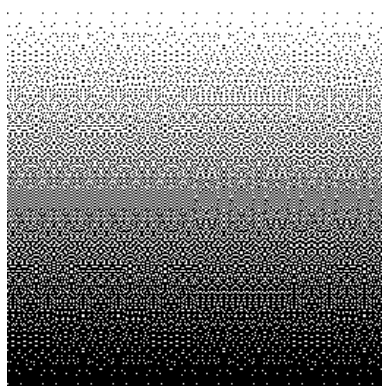

(b)

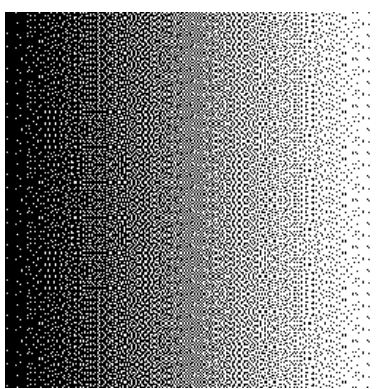

(d)
Figure 7. A comparison between the scanning orders in the IGS quantization: Results from the vertical ramp image by (a) Raster scanning and by (b) Hilbert scanning; results from the horizontal ramp image by (c) Raster scanning and by (d) Hilbert scanning. All the images are displayed at a resolution of 200 pixels per inch (PPI).

halftones achieved by the IGS halftoning. Assuming halftone images produced by EDF-FS to be of standard quality, we compare the IGS halftone images with the EDF-FS ones, and then, evaluate a halftone quality of the IGS produced images from the amount of perceptible difference. Thus, a subjective quality of multi-level halftones produced by each IGS method will be evaluated. Also, the printing resolution is to be taken into account as a condition of evaluation.

For measuring the above image difference by subjecttive evaluations of human observers, the following experiment was conducted by using a category-judgment method [6]: In preparation, the halftone images each produced from the same source image by EDF-FS and the three IGS methods with a given $N$ were printed on the same photographic paper with a photo printer at a printing resolution of 400 dots per inch. Comparing with the EDF-FS halftone image on the paper, the observers evaluated each IGS halftone image from the degree of perceptible difference between them on the scale listed in Table 1.

Each image was evaluated by 21 observers. All the observers, who were in their twenties, were unfamiliar with any of the methods. The result of evaluating each IGS image is presented by computing a mean value, which is generally referred to as a Mean Opinion Score (MOS), from the collected values.

\subsection{Experimental Result and Discussion}

Figures 8 (a) and (b) show the variations of MOS with $N$ for the 200-PPI halftone images of the source image Barbara and Lena, respectively, for each IGS method separately. The error bars in the figures illustrate the 0.95-confidence intervals of the respective MOS values.

Figure 9 shows those for the 100-PPI halftone images. In addition, Figure $\mathbf{1 0}$ presents a comparison between EDF-FS halftone images and IGS-HIL halftone images of a resolution of 200 PPI for each $N$.

These figures demonstrate the properties of $\mathrm{N}$-bit halftones by the IGS quantization. The values of MOS increase with increasing $N$ in any IGS method, and this means that the IGS halftone image looks closer to the EDF-FS halftone image, and consequently, to the source image as more levels are used in halftoning. We can observe such effect of level multiplicity in Figure 10. Also, the methods IGS-RAS and IGS-HIL have almost the same increasing properties with $N$. However, the curves of IGS-RAS show more fluctuation with $N$ than those of

Table 1. Scale used in subjective evaluations.

\begin{tabular}{cc}
\hline Value & Description \\
\hline 3 & Difference is hardly perceptible. \\
2 & Difference is perceptible but negligible. \\
1 & Difference is definitely observed. \\
\hline
\end{tabular}

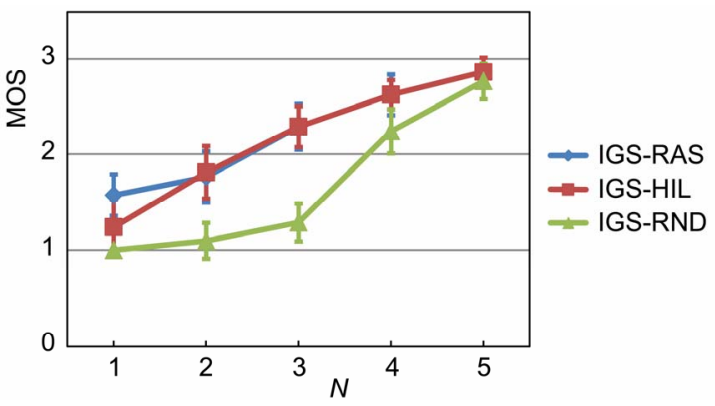

(a)

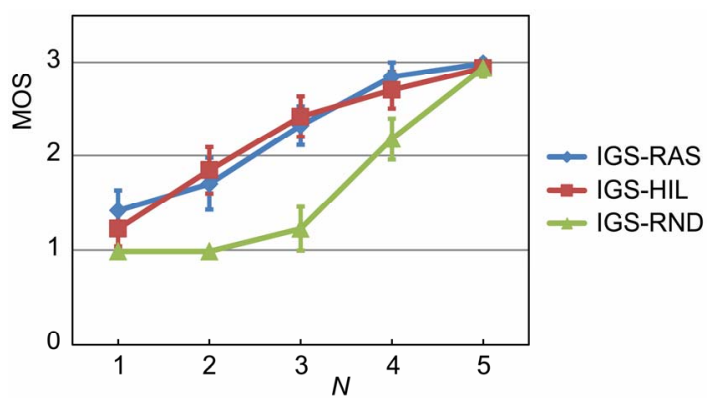

(b)

Figure 8. Measurements of subjective quality of $N$-bit halftone images printed at 200 PPI: Result from (a) The source image Barbara; (b) Lena. 


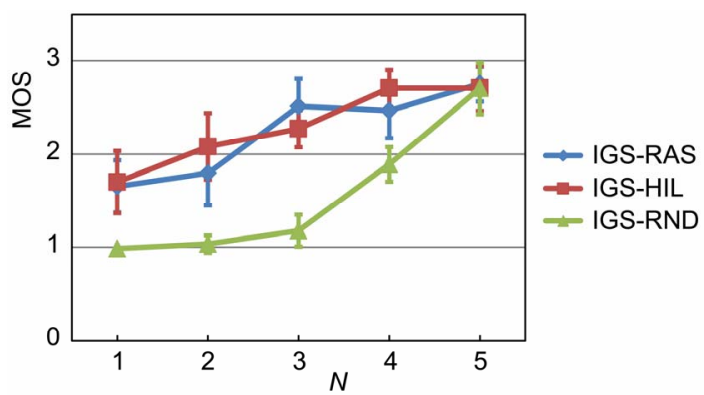

(a)

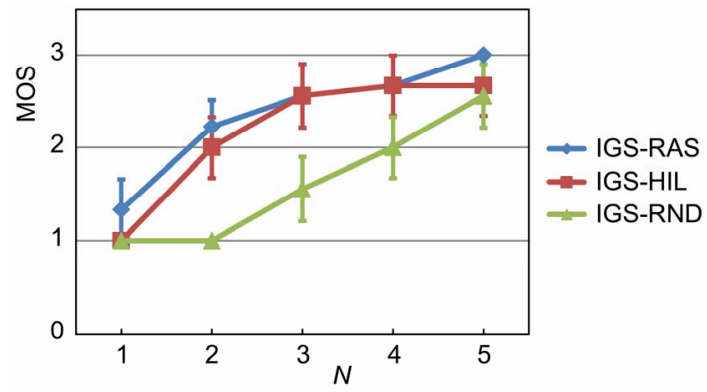

(b)

Figure 9. Measurements of subjective quality of $\mathrm{N}$-bit halftone images printed at 100 PPI: Result from (a) the source image Barbara; (b) Lena.

IGS-HIL. The fluctuation is probably due to the dependence of IGS-RAS on scanning direction.

Let us suppose that an IGS halftone image with MOS over 2 can be used instead of the corresponding EDF-FS halftone image. Then, as Figures 8 and $\mathbf{9}$ show, for IGSHIL, the halftones of 3 or more bits $(N \geq 3)$ achieve the MOS values larger than 2 including those in the 0.95 -confidence interval. As for IGS-RND, at least 4 bits are necessary to achieve such MOS values.

In addition, the visual quality of multi-level halftone images including those by EDF-FS was evaluated by subjective testing. In the experiment, the observers chose one or more images that look closest to the source image in the five $N$-bit halftone images produced by the respective methods of IGS-RAS, IGS-HIL, IGS-RND, EDF-FS and EDF-JJN. By using four different set of a printed source image and the five printed halftone images, the ratios that the halftone images by each method were chosen in all the sets were calculated.

Figure 11 shows the variation of the ratio of each method with $N$. This experimental result indicates that EDF-FS achieves the best visual quality in the five methods for any $N$-bit multi-level halftones and also that both IGS-RAS and IGS-HIL methods increase the capability of achieving the best $N$-bit multi-level halftone quality with an increase in $N$.

\section{Conclusions}

In halftoning by the IGS quantization, the resulting half

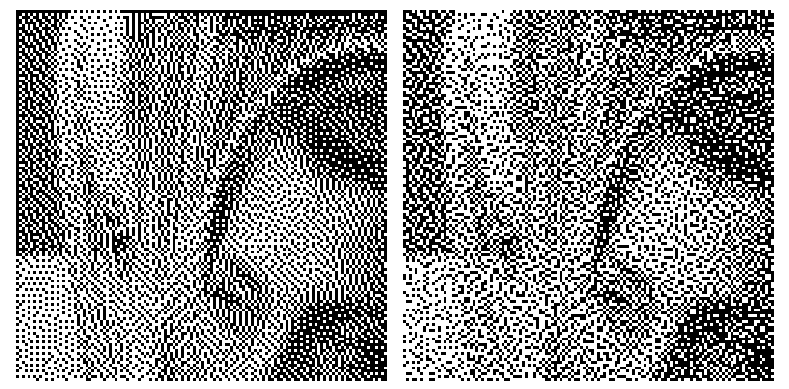

(a)
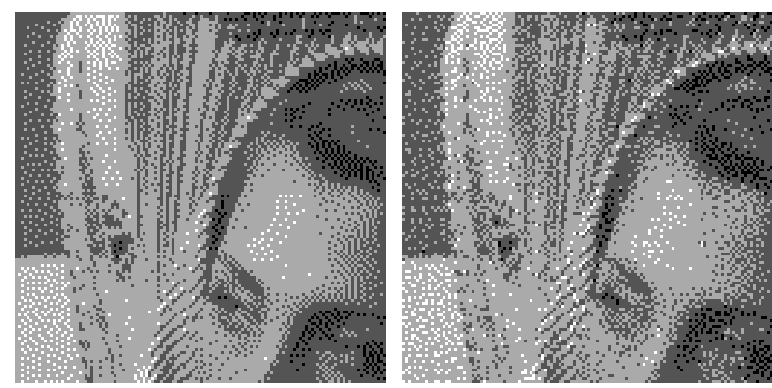

(b)
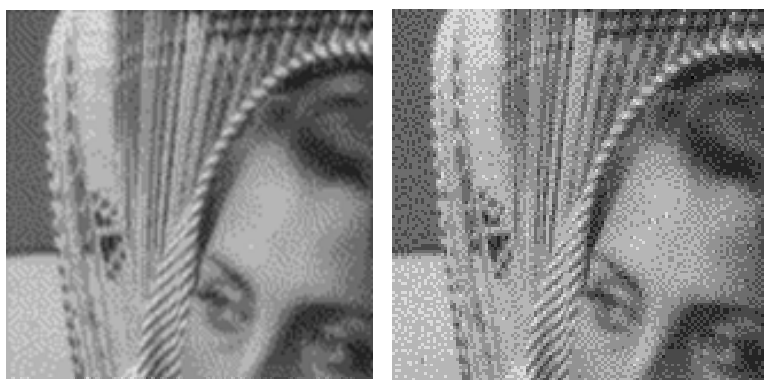

(c)
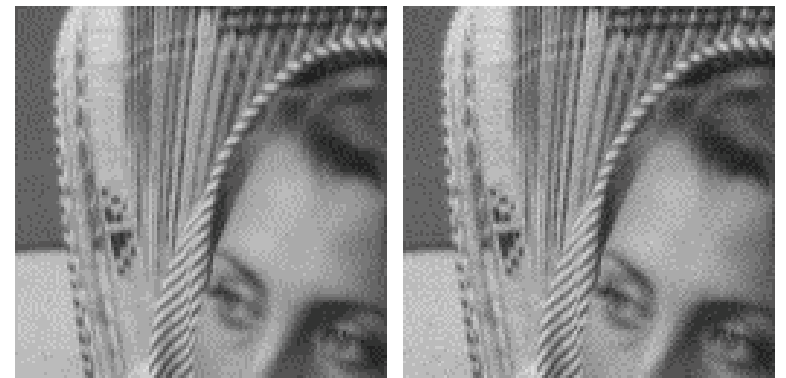

(d)

Figure 10. $N$-bit Halftone images of the source image Barbara by EDF-FS (left column) and by IGS-HIL (right column), which are zoomed parts of the respective images: (a) $N=1$; (b) $N=2$; (c) $N=3$; (d) $N=4$.

tones are subject to the scanning order in a source image. Accordingly, geometric patterns are likely to be produced in areas of smooth gray levels. Such artifacts degrade the image quality, in particular in bi-level $(N=1)$ halftones.

Such a directional relation between a level gradient and the scanning path can be reduced by using a complicated path such as the Hilbert path. The experimental 


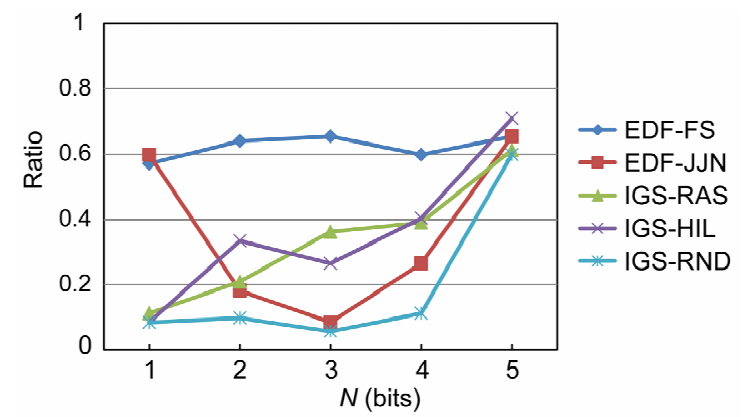

Figure 11. Experimentally measured ratios of each $\mathrm{N}$-bit halftone image looking closest to the source image between those generated by the five methods.

result demonstrated that the Hilbert scanning makes the additional signals for the IGS quantization independent of level gradients, and also, increases their randomness. Consequently, the IGS halftone image by Hilbert scanning looks a little grainier with high-frequency signal components somewhat degraded than that by simple raster scanning.

Bi-level halftone images by IGS-HIL probably look worse than those by the error diffusion scheme. Using multi-level halftones improves visual quality of IGS-HIL halftone images. By using three or more bits, that is, 8 or more levels for a halftone level, the IGS-HIL images look comparable to the EDF-FS images.

Such 8-level halftone images are supposed to be printed at a resolution of one pixel per dot with a printer of a capability of eight levels per dot. Otherwise, they can be printed with a bi-level printer, by expressing one 8-level halftone pixel by a set of bi-level dots by using another halftoning method such as clustered-dot ordered dithering [7]. For instance, a set of $3 \times 3$ bi-level dots can represent a 10-gray scale pixel.

The IGS quantizing is one of what we call point processing. Hence, the operation of the quantization is obviously simpler than that of the convolution of filtering in the error diffusion. Although the IGS quantization may be an old-fashioned method, the operational simplicity as well as the above quality comparability to EDF-FS makes the IGS multi-level halftoning useful in recent image output devices.

\section{REFERENCES}

[1] R. Ulichney, “Digital Halftoning,” MIT Press, Cambridge, 1993.

[2] R. C. Gonzalez and R. E. Woods, "Digital Image Processing,” Addison-Wesley, Boston, 1993.

[3] N. D. Venkata, B. L. Evans and V. Monga, "Color Error Diffusion Halftoning,” IEEE Signal Processing Magazine, Vol. 20, No. 4, 2003, pp. 51-58.

http://dx.doi.org/10.1109/MSP.2003.1215231

[4] R. W. Floyd and L. Steinberg, "An Adaptive Algorithm for Spatial Grayscale,” Proceedings of the Society of Information Display, Vol. 17, No. 2, 1976, pp. 75-77.

[5] J. F. Jarvis, C. N. Judice and W. H. Ninke, "A Survey of Techniques for the Display of Continuous-Tone Pictures on Bilevel Displays," Computer Graphics and Image Processing, Vol. 5, No. 1, 1976, pp. 13-40. http://dx.doi.org/10.1016/S0146-664X(76)80003-2

[6] A. N. Netravali and B. G. Haskell, "Digital Pictures," Plenum, 1988.

http://dx.doi.org/10.1007/978-1-4684-1294-9

[7] P. G. Roetling and R. P. Loce, "Digital Halftoning,” In: E. R. Dougherty, Ed., Digital Image Processing Methods, Marcel Dekker, New York, 1994, pp. 363-413. 